\title{
The FDI World Dental Federation web-site
}

\author{
Wil JM van der Sanden and Dirk G Mettes \\ University Medical Center Nijmegen, College of Dental Sciences, Nijmegen, \\ The Netherlands
}

The FDI World Dental Federation web-site contains information about the organisation itself, about congresses and dental manufacturers, and has a special area for dental professionals. The authors feel that this web-site is an interesting and valuable electronic portal for general dental practitioners and dental researchers, as well as for national dental organisations. The site contains a tremendous amount of information. Some recommendations are offered to further improve this informative and well-constructed web-site.

Evidence-Based Dentistry (2002) 3, 81-83. doi:10.1038/sj.ebd.6400127

\section{Introduction}

Looking for the best available information on dentistry on the Internet, you may find it helpful to visit the FDI site. The FDI World Dental Federation is an independent, international organisation for dental professionals. The activities of the federation cover most aspects of dentistry both on a national and international level.

The FDI web-site (http://www. fdiworldental.org/) has been constructed at the new FDI World Dental Federation headquarters in FerneyVoltaire, France. For this article, only the 'Professional Resources' area was reviewed to assess the accessibility and content of this site (for these pages, go to the URL http://www.fdiworldental. org/resources/index.htm, or press the green button on the home-page).

\section{Layout}

The dominant colour pattern of 'Professional Resources' - black text on a white background with coloured upper-case titles for the subdivisions - results in a balance between simplicity and visual impact. The blue, red and green screen buttons allow easy navigation to clearly-defined headings and topics. Clear and straightforward, as most general dental practitioners would prefer. There are keyword search facilities available, which is important to stimulate inexperienced users or for those who need to locate information quickly. The identification of the site sources and the contact addresses of the head office are all mentioned. The date of the last revision of the web-site, in contrast, was not easy to find, but consecutive visits over an evaluation period of almost 3 weeks suggested that the site is updated on a regular basis. As the site is still under construction, some links to other sites were not operational at the time of evaluation.

\section{Professional resources}

The declared aim of the 'Professional Resources' section is, 'to disseminate policies, standards and information related to all aspects of oral health care especially for individual members and members' associations' (see Figure 1). A site search is available. The Professional Resources site can be viewed in seven languages (just use the flag button) and is subdivided into five sections. These sections are easy to activate with hyperlinks to the 'Guidelines database', 'Dental journals on WWW', 'Continuing education courses', 'Inquiries dental science' and 'Emerging technologies'.

\section{Guidelines database}

The database contains an overview of references and links to national and international guidelines and statements, position papers, proceedings, systematic reviews and meta-analyses. In seven subdivisions all aspects of professional oral health care are covered. All subdivisions are divided into the three categories 'world', 'FDI' and 'FDI statement' (if available). Not all links to these subjects are active. The title of this section is somewhat confusing, as very few clinical practice guidelines are contained, whereas most of the documents are related to statements and position papers.

How can we use this site? The following example will illustrate.

My patient wants to know whether his impacted, symptom-free wisdom teeth should be removed, the so-called prophylactic removal of asymptomatic third molars. Using the keywords 'third, molar, removal, prophylactic', this search resulted in 53 hits. The hits can be sorted by date. The first two hits did match the question about indication/therapy, and linked to a publication and an evidence-based report, respectively. The hyperlink to the level of evidence ('Evidence strength') was inactive, however. The third hit was irrelevant; the fourth hit 


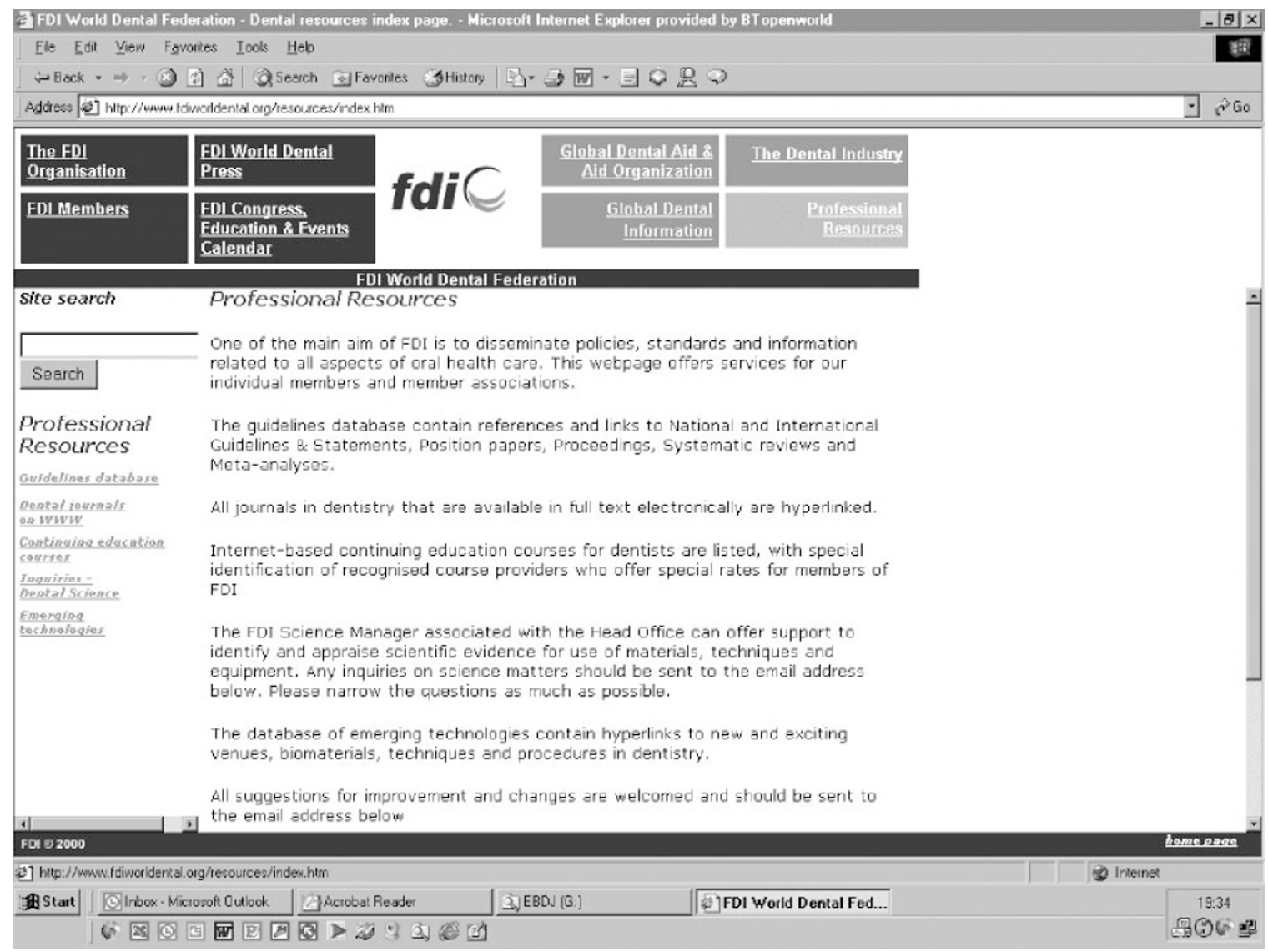

Figure 1

could not be opened, whereas the fifth and sixth hits were related to psychomotor skills, ie, surgical procedures without decision-making. The seventh hit linked to oral surgery guidelines, which also matched the predefined criteria. Not all links on the guidelines database site are active yet, and the quality ('Evidence strength') of the guidelines is not mentioned, which is a pity.

A second search for clinical practice guidelines for the daily use in the dental practice on a patient level, using the keywords, 'clinical, practice, guidelines resulted in abut 340 links to all kinds of guideline development procedures, statements procedures, position papers and other quality instruments on different national levels. It is fun to browse through some of the material, but it is clearly too much work to study everything.
The busy general dental practitioner will not have the time to extract the appropriate information for the specific clinical decision-making process out of these extensive lists of differently qualified papers. Some more structure would be welcome.

\section{Dental journals on the WWW}

A series of international published dental journals that are available electronically in full text are accessible via hyperlinks. Using simple buttons you can scroll through and reach more than 70 international dental journals available on the Web. Most of these offer free access to titles or abstracts of manuscripts, but do not permit free access to the full text articles, unless you subscribe. Nevertheless, this facility provides a quick and easy way to find information.

\section{Continuing education courses}

This page offers a large list of continuing education courses for dental professionals. The hypertext links are clear and of interest especially for general dental practitioners. In testing, the majority of the links could be activated successfully. The 'Continuing education course' hyperlink offers an extensive survey for all kind of courses, has a user-friendly buttonsystem with nice and easy links to 60 homepages of different course providers, most of them located in the US or the UK (see Table 1).

\section{Inquiries dental science}

At this page, the FDI Science Manager offers support to identify and appraise scientific evidence for the use of materials, techniques and equipment.

This section offers a list of frequently asked questions (FAQ), divided in 
Table 1 Continuing education courses as mentioned on the FDI-website

\begin{tabular}{ll}
\hline FDI-endorsed providers $(n=2)$ & Other providers $(n=58)$ \\
\hline USA (1) & Academic institutions international (2) \\
UK (1) & Academic institutions US/Canada (18) \\
& Commercial international (4) \\
& Commercial US/Canada (24) \\
& Professional associations, other organisations (10)
\end{tabular}

categories. If you choose a clinical topic of interest and find it in the list of clinical questions, you are sent to the literature reference, research design and levels of evidence ('Best evidence'). The hyperlink to 'Evidence strength', however, cannot be activated. The hyperlink to 'Appraisal' is mostly inactive, as this is still under preparation. In a few cases you will find a critical appraisal of the best-evidence literature, without a remark about whose statement it is and when it was made. Nor could we find a reference for the so-called 'levels of evidence'.

\section{Emerging technologies}

This page contains hyperlinks to new and exciting venues, biomaterials, techniques and procedures in dentistry. Besides a disclaimer, stating that there exists no direct connection between the federation and the firms mentioned, you will find about 15 links to companies and products which offer new technologies and materials in diagnostic and therapeutic dentistry.
For example, a Dutch company promotes 'quantitative light-induced fluorescence' for caries diagnostic procedures and a well-known company from the US introduces a new alternative to the injection of anaesthetics in pain control. The dental professional who wants to be updated with new and emerging technology in dentistry is encouraged to add this page to his 'Favourites'.

\section{Recommendations}

The FDI site clearly endeavours to support its statements with the evidence from literature. We would recommend that this is done in a more structured manner, eg, by creating a site specially devoted to evidence-based clinical practice guidelines, describing why and for whom they are developed, how systematically the developing process was carried out, who is involved in their development and so on. This synthesis between clinical expertise and best available evidence united in clinical practice guidelines for the use of the dental professional could encourage dental professionals on a national and world-wide level, and also motivate them to make clinical decisions based on valid information extracted from the available evidence.

\section{Conclusion}

The 'Professional resources' part of the FDI web-site is well-built and quite informative for the general dental practitioner as well as for researchers in dentistry. Improvements are suggested for the content of the 'Guideline database' and 'Inquiries dental science' sections to stimulate links between evidence and clinical decision-making in daily practice in an easy and straightforward manner. The general dental practitioner will definitely appreciate this. The authors recommend general dental practitioners, within whose scope it is to obtain good and reliable information, to visit this FDI web-site. Those who do so will find it rewarding, and will certainly add the site address to their personal list of 'Favourites' for further visits.

\section{Author's note}

This web-site was reviewed over the period 2-22 June 2002. The content and layout may have changed by the time of publication. 